\title{
Los mediosaudiovisuales del centro-sudeste bonaerense en el período 1983-2004
}

\author{
María Eugenia Iturralde \\ eugeniaiturralde@gmail.com.ar
}

CONICET. Centro de Estudios de Teatro, Educación y Consumos Culturales, Facultad de Arte, Universidad Nacional del Centro de la Provincia de Buenos Aires.

\author{
Fecha de finalización del trabajo: 26 de febrero de 2020 \\ Recibido: 27 de febrero de 2020 \\ Aceptado: 11 de junio de 2020 \\ DOI: https://doi.org/10.26422/aucom.2020.0901.itu
}

\section{Resumen}

En el presente artículo se analiza la implementación de políticas de comunicación a nivel nacional y su incidencia en ciudades medias del centro-sudeste de la provincia de Buenos Aires. Se tomaron específicamente los casos de Azul, Olavarría y Tandil en el período comprendido desde la asunción de Raúl Alfonsín a la presidencia de la nación (1983) hasta la presentación de los 21 Puntos Básicos por una Comunicación Democrática (2004).

La etapa abordada se inscribe en el contexto de profundización de los procesos iniciados con la caída de la matriz estado-céntrica (Cavarozzi, 2002, p. 66), momento en el que desde el plano económico se instauró un sistema de libre mercado que consistió en abrir el comercio interno a la competencia exterior. En lo que respecta a los medios de comunicación, diversos estudios refieren a la existencia de procesos de concentración y extranjerización de la propiedad de estos. El análisis nos deja ver que los medios de las ciudades antes mencionadas siguieron el ritmo sostenido por la re-regulación impulsada a nivel nacional. Las experiencias locales no muestran avances de proyectos sin fines de lucro, sino una fuerte preeminencia de actores del sector privado como prestadores y algunas iniciativas públicas sostenidas por instituciones que se encuentran dentro de la órbita de la Iglesia católica argentina. También se observa el surgimiento de grupos de medios locales, la integración de proyectos radicados en Olavarría a un multimedio de la Ciudad Autónoma de Buenos Aires y la extranjerización de la propiedad de los servicios de cable.

En la indagación se optó por una estrategia metodológica de carácter cualitativo. Para la producción de datos se articularon diferentes técnicas y procedimientos: entrevistas abiertas, análisis de documentos y de publicaciones de medios de comunicación consultadas en archivos de las ciudades analizadas. Estas técnicas de producción de información empírica dieron lugar a la interacción y articulación entre datos primarios y datos secundarios. 
Palabras clave: políticas de comunicación, medios audiovisuales, ciudades medias, provincia de Buenos Aires.

\title{
Audiovisual media in the south central area of Buenos Aires Province from 1983 to 2004
}

\begin{abstract}
In this article, we will analyze the implementation of communication policies at the national level and their effect on medium-sized cities in the south central area of Buenos Aires Province. We will specifically look at the cases of Azul, Olavarría, and Tandil during the period stretching from Raúl Alfonsín's swearing-in as president of Argentina (1983) to the drafting of the 21 Essential Points for Democratic Communication (2004).

This era was marked by the entrenchment of processes initiated by the fall of the state-centered matrix (Cavarozzi, 2002, p. 66), which, from an economic perspective, saw the establishment of a free market system that opened domestic trade to foreign competition. In regard to communication media, as various studies have pointed out, their ownership underwent processes of concentration and foreign takeover. Our analysis shows how media outlets in the aforementioned cities kept pace with this nationwide trend towards re-regulation. Local examples reveal no advances in non-profit projects, but rather the prominent role of players from the private sector as service providers and public initiatives backed by institutions in the orbit of the Argentine Catholic Church. We also observed the emergence of local media groups, the integration of projects based in Olavarría to a multimedia company in the Autonomous City of Buenos Aires, and the foreign takeover of cable services.

For our study, we opted for a qualitative methodological approach. To produce our data, we combined different techniques and processes: open-ended interviews along with an analysis of documents and publications by different communication media stored in archives in the aforementioned cities. These techniques for empirical information gathering led to the interaction and articulation of primary and secondary sources.
\end{abstract}

Keywords: communication policies, audiovisual media, media cities, Buenos Aires province

\section{Meios audiovisuais do centro da província de Buenos Aires no período 1983-2004}

\section{Resumo}

Neste artigo, analisamos a implementação de políticas de comunicação em cidades do Centro da província de Buenos Aires, Argentina; os casos de Azul, Olavarría e Tandil foram tomados especificamente no período desde que Raúl Alfonsín teve assumido até a presidência da Nação - em 1983 - até a apresentação dos 21 Pontos Básicos pra uma Comunicação Democrática - no ano 2004 .

A etapa analisada faz parte do contexto de aprofundamento dos processos iniciados com a queda da matriz centrada no Estado, quando desde o nível econômico foi estabelecido um sistema de livre mercado que consistia em abrir o comércio interno à concorrência estrangeira. No caso da mídia, vários estudos referem-se à existência de processos de concentração e estrangeirização de sua propriedade. No caso de experiências locais registradas, elas mostram uma forte proeminência de atores do setor privado como fornecedores e algumas iniciativas públicas sustentadas por instituições que estão dentro da órbita da Igreja Católica Argentina. 
Austral Comunicación

Volumen 9, número 1 (Junio de 2020): 93-119. ISSN 2313-9129

\begin{abstract}
$\mathrm{Na}$ análise, foi escolhida uma estratégia metodológica qualitativa. Para a produção dos dados, foram articuladas diferentes técnicas e procedimentos: entrevistas abertas, análise de documentos e publicações na mídia, consultadas em arquivos das cidades analisadas. Essas técnicas empíricas de produção de informações, resultaram na interação e articulação entre dados primários e secundários.

Palavras chave: políticas de comunicação, mídia audiovisual, cidades médias, província de Buenos Aires.
\end{abstract}

\title{
Introducción
}

El presente trabajo forma parte de una investigación que abarca un extenso período de tiempo, por eso, con el objetivo de presentar los resultados, realizamos una división en diferentes etapas que permiten abordar de manera procesual la implementación de políticas públicas sobre medios de comunicación audiovisuales y su incidencia en ciudades medias del centro-sudeste bonaerense. La primera etapa abarca de 1922 a 1983, momento histórico marcado por seis golpes de Estado de características variadas y también por el dictado de tres normativas integrales sobre medios audiovisuales (solo una fue sancionada por un Gobierno constitucional). Es un período de tiempo muy amplio que contiene, en términos históricos, cortes político-institucionales sumamente relevantes, pero que fue delimitada respondiendo a criterios propios de la investigación con relación a las políticas de comunicación.

Señalamos un segundo momento que comprende desde que Raúl Alfonsín asumió la presidencia -dando fin a la última dictadura militar en 1983- hasta la presentación de los 21 Puntos Básicos por una Comunicación Democrática (2004). En este período, organizaciones de la sociedad civil sostuvieron el reclamo para que se aprobara una ley de radiodifusión de la democracia, pero el Estado no avanzó en ese sentido, sino que sancionó normativas parciales y modificatorias que posicionaron al mercado de manera predominante en el sector y dieron lugar -específicamente durante la década del 90- a procesos como la concentración y extranjerización de la propiedad de los medios audiovisuales (Mastrini y Becerra, 2001).

En la tercera etapa (2004 a 2015), en términos generales se observa que el Estado adoptó un nuevo rol $^{1}$ respecto a los períodos anteriores, ya que reivindicó acciones políticas de algunas organizaciones de la sociedad civil, sancionó la Ley 26522 de

\footnotetext{
La primera década del siglo XXI está marcada por el triunfo electoral de diversas fuerzas políticas en gran parte de los Estados de América Latina, las cuales suelen ser denominadas como "progresistas". Badillo, Mastrini y Marenghi (2015) señalan tres rasgos comunes que poseen estos Gobiernos latinoamericanos del nuevo siglo. En primer término, destacan que tienen voluntad de recuperar el rol regulador y productor del Estado, en segundo lugar, la preocupación (retórica y con frecuencia sustancial) por la redistribución del ingreso y la justicia social, y, por último, la reivindicación de la sociedad civil como espacio de acción política basada en la promoción de mecanismos de participación democrática.
} 
Servicios de Comunicación Audiovisual y se posicionó como emisor y gestor de la comunicación (Becerra, 2014).

Con el objetivo de analizar los procesos antes mencionados, tomamos la propuesta teórico-metodológica expresada en publicaciones recientes, en las cuales se analizan las políticas de comunicación entendiéndolas como políticas públicas (Bizberge, 2015; Califano, 2015; Hernández Prieto y de la Peña Pérez Alaejos, 2017; Marino, 2017a). Desde esta perspectiva, se toman conceptualizaciones provenientes de la ciencia política sobre políticas públicas (Oszlak, 2003; 2007; Oszlak y O’Donnell, 1995) para el análisis específico de los medios de comunicación.

Asimismo, se adoptó una estrategia metodológica de carácter cualitativo. En la producción de datos se emplearon diferentes procedimientos y métodos para la obtención de información empírica. Se realizaron 12 entrevistas abiertas, ${ }^{2}$ se efectuó en 2015 un registro de medios operativos en Azul, Olavarría y Tandil -materializados en tablas de información organizada $-{ }^{3}$ y se revisó un corpus jurídico de 98 normativas recuperadas del sitio web del Boletín Oficial de la República Argentina. ${ }^{4}$ Para el análisis se tomaron los siguientes referentes empíricos: normativas, instituciones intervinientes, documentos, actores político-institucionales, políticas de comunicación y publicaciones periodísticas consultadas en la Hemeroteca Juan Miguel Oyhanarte de Azul, la Biblioteca Rivadavia de Tandil y el archivo del licenciado Jorge Arabito en Olavarría. ${ }^{5}$ Estas técnicas de producción de información empírica dieron lugar a la interacción y articulación entre datos primarios y datos secundarios.

\section{Políticas públicas y políticas de comunicación}

\footnotetext{
Se efectuaron entre noviembre de 2011 y octubre de 2017. Se entrevistó a un investigador de medios regionales, a tres trabajadores de la Autoridad Federal de Servicios de Comunicación Audiovisual (AFSCA), a una radiodifusora sin fines de lucro, a dos radiodifusores con fines de lucro, a dos coordinadores de radios públicas, a un trabajador de medios de comunicación, a un exfuncionario municipal de Olavarría y a una trabajadora del municipio de Tandil.

3 Las tablas fueron confeccionadas desde mayo hasta diciembre de 2015. Los datos consignados se extrajeron de relevamientos efectuados por la AFSCA en Azul, Olavarría y Tandil, del sitio web de la AFSCA, de la escucha de la amplitud modulada y de la frecuencia modulada en diferentes horarios del día en las cabeceras de los partidos, consulta a informantes clave, de publicaciones de medios de comunicación, del Boletín Oficial de la República Argentina, del sitio web NIC Argentina y de búsquedas en internet.

${ }_{4}$ Obtenidos del sitio web www.boletinoficial.gob.ar, se consultaron los siguientes números del Boletín Oficial en versión impresa digitalizada, publicados entre el 19 de septiembre de 1980 y el 7 de junio de 2003: 24506, 24614 $24631,24891,25394,25409,26007,26131,26467,26659,26702,26725,26726,26776,26790,26995,27025,27081$, 27133, 27214, 27254, 27406, 27419, 27428, 27469, 27480, 27589, 27633, 27814, 27938, 27963, 28057, 28117, 28304 $28304,28321,28415,28463,28497,28500,28518,28565,28591,28673,28689,28824,28828,28862,28975,29021$ 29049, 29059, 29088, 29171, 29214, 29233, 29238, 29253, 29294, 29300, 29310, 29357, 29425, 29 468, 29476, 29478 29488, 29357, 29425, 29468, 29476, 29478, 29488, 29490, 29492, 29538, 29539, 29577, 29650, 29687, 29695, 29760, 29777, 29781, 29782, 29799, 29801, 29839, 29927, 30089 y 39186.

5 El licenciado Jorge Arabito posee un extenso archivo que incluye producciones de medios de comunicación gráficos y audiovisuales- de la región centro-sudeste bonaerense, generado a partir de su relevamiento personal y de distintos proyectos radicados en la Facultad de Ciencias Sociales de la Universidad Nacional del Centro de la Provincia de Buenos Aires.
} 
Austral Comunicación

Volumen 9, número 1 (Junio de 2020): 93-119. ISSN 2313-9129

Los análisis de las políticas de comunicación suelen involucrar teorías y herramientas provenientes de la ciencia política y del campo de la comunicación, los cuales permiten examinar el proceso de formulación de políticas públicas referidas a medios. En esta investigación se entiende a las "políticas de comunicación" como políticas públicas, por eso tomamos la propuesta de Oscar Oszlak y Guillermo O’Donnell (1995) para establecer un marco analítico que permita abordar el tema de nuestro interés.

La agenda social problemática involucra tres tipos de actores: instituciones estatales, proveedores del mercado y organizaciones de la sociedad civil. Bernadette Califano (2015) propone para el abordaje de políticas de medios analizar las acciones u omisiones del Estado y la intervención de diversos actores (grupos sociales, fuerzas políticas, individuos) con capacidad de negociar en el ámbito político, intentando favorecer sus intereses. La autora considera que el Estado procesa los poderes que emergen de la sociedad, los materializa en acciones, omisiones e impactos y en diversas políticas públicas. También es necesario tener en cuenta que las políticas públicas involucran diferentes dependencias que se organizan en instancias municipales, provinciales y nacionales, y que a veces persiguen objetivos contrapuestos. El Estado es un actor decisivo en el diseño e implementación de políticas de comunicación, pero también es prudente decir que no es el único. En estas políticas también intervienen entidades no gubernamentales y privadas que persiguen intereses particulares.

José Luis Exeni (2010) considera que el análisis de las políticas públicas de comunicación requiere de una definición de "Estado", de la identificación de los actores sociales, políticos e institucionales involucrados y su grado de participación y del reconocimiento de la conflictividad entre el sector público y el privado.

De acuerdo con la propuesta de Exeni (2010), tomamos las palabras de Guillermo O’Donnell (2010) cuando sostiene que el Estado:

\begin{abstract}
Es una asociación con base territorial, compuesta de conjuntos de instituciones y de relaciones sociales (la mayor parte de ellas sancionadas y respaldadas por el sistema legal de ese estado) que normalmente penetra y controla el territorio y los habitantes que ese conjunto delimita. Esas instituciones reclaman el monopolio en la autorización legítima del uso de la coerción física y normalmente tienen, como último recurso para efectivizar las decisiones que toman, supremacía en el control de los medios de esa coerción sobre la población y el territorio que el estado delimita (p. 76).
\end{abstract}

Esta definición contribuye a pensar sobre el conjunto de poderes que implican el surgimiento y el funcionamiento de un Estado. Este, por un lado, condensa y procesa poderes que emergen de la sociedad y, por otro, crea poderes propios que le permiten implementar diversas políticas públicas que atiendan las demandas de la comunidad. Es necesario señalar que el Estado aborda algunas problemáticas, así como también suele omitir otras que integran la agenda social (O'Donnell, 2010). Las instituciones del Estado articulan las relaciones sociales, a la vez que ejercen dominación política. 
María Eugenia Iturralde

Los medios audiovisuales del centro-sudeste bonaerense en el período 1983-2004

Extraen recursos de la comunidad con el objetivo de brindarle bienes y servicios, cuya prestación es considerada propia de su rol frente a la sociedad.

Como mencionamos antes, además del Estado, los actores sociales involucrados son el mercado y la sociedad civil. El primero es visto como un ámbito de participantes movidos, fundamentalmente, por el lucro. Dentro de la categoría de sociedad civil suelen ubicarse las organizaciones no gubernamentales y, más genéricamente, las organizaciones y movimientos sociales.

Con respecto a la conflictividad a la que se refiere Exeni (2010), es necesario señalar que considerar la información y la comunicación como asuntos vinculados a las políticas públicas es una propuesta históricamente resistida por algunos sectores. El empresariado rechaza la regulación de los medios de comunicación argumentando que el Estado debe ocuparse de áreas menos "rentables", como la salud y la educación (Graziano, 1988).En América Latina, por lo general, desde el sector privado de medios se sostiene la concepción liberal clásica de "libertad de expresión", que concibe a los Gobiernos como un peligro (Becerra, 2015). De acuerdo con estos posicionamientos, el Estado no tiene que garantizar servicios que resultan redituables para el mercado y, además, su intervención se constituye en un obstáculo para el desarrollo comercial.

\section{Leyes y radiodifusión en la Argentina del siglo XX}

En el siglo XX, se sancionaron tres normativas integrales sobre servicios audiovisuales: en 1953 el Senado de la Nación aprobó la Ley 14241; en 1957 se dictó el decreto-ley 15460 -bajo el Gobierno de facto de Pedro Eugenio Aramburu-; y en 1980, el decreto-ley 22285. A los fines de este trabajo, nos vamos a detener en esta última, ya que es el marco normativo vigente durante el período analizado. El decreto-ley 22285 de Radiodifusión fue el instrumento de regulación de los servicios audiovisuales que más tiempo estuvo en vigencia en la historia del Estado argentino; se sancionó en 1980, se reglamentó en 1981 y perdió vigencia en 2009 con la aprobación de la Ley 26522 de Servicios de Comunicación Audiovisual.

A mediados de la década del 70, la Argentina atravesó una profunda crisis económica y fiscal -acompañada de conflictos distributivos y enfrentamientos político-ideológicos intrapartidarios- que decantó en una reestructuración tanto política como económica. Marcelo Cavarozzi (2002) señala ese momento histórico como el de la caída de la matriz estado-céntrica. ${ }^{6}$ El 24 de marzo de 1976, se produjo un feroz ataque a las instituciones democráticas en Argentina. Las Fuerzas Armadas

\footnotetext{
6 Cavarozzi (2002) analiza la evolución del Estado argentino y refiere al período que va de 1930 a mediados de la década del 70 como matriz estado-céntrica (MEC). El autor señala que durante ese período primaron los principios del Estado de bienestar y se asumió una fuerte presencia estatal en la regulación de la economía.
} 
dieron un golpe de Estado y de ese modo se inició el autodenominado "Proceso de Reorganización Nacional"?

En materia de políticas de comunicación, el Gobierno de facto puso a los medios de comunicación al servicio del disciplinamiento social. Llevó adelante políticas de desinformación, censura y manipulación mediática (Postolski y Marino, 2006). La toma ilegítima del poder durante la última dictadura significó la disolución del Congreso, la imposición de la Ley Marcial y la gobernanza por decreto. En este contexto fue que se sancionó el decreto-ley 22285 de Radiodifusión.

Funcionarios del Poder Ejecutivo Nacional (PEN), asesorados por las organizaciones patronales de la radiodifusión, diseñaron este decreto-ley, cuyo contenido contemplaba el control ideológico de interés del Gobierno de facto y el fin de lucro perseguido por el empresariado (Postolski y Marino, 2006). El control se expresó en los requisitos para acceder a las licencias de radiodifusión, que podían ser otorgadas a personas físicas o jurídicas con fines de lucro que exclusivamente prestaran y explotaran servicios de radiodifusión. Las empresas solo podían contar con veinte personas físicas como socias, el ingreso de capital extranjero estaba prohibido, las empresas de medios gráficos no podían acceder a licencias de radiodifusión y se prohibía la emisión en red.

Las autoridades de aplicación de la ley eran el Comité Federal de Radiodifusión $(\text { COMFER })^{8}$-a cargo del control cultural, artístico, legal, comercial y administrativoy la Secretaría de Comunicaciones, encargada de realizar tareas técnicas vinculadas a la adjudicación y al control de operación de las licencias. Las estaciones sin autorización legal eran consideradas clandestinas y, ante su identificación, correspondía el decomiso o incautación de los bienes que les estuvieran afectados. El texto normativo fijaba la regulación de la televisión y la radio abiertas y de los servicios complementarios: televisión por cable, antenas comunitarias y televisoras codificadas. Al decreto-ley de Radiodifusión le siguió la sanción del Plan Nacional de Radiodifusión -conocido como PLANARA-, cuyo Documento Técnico Básico estableció las frecuencias disponibles.

En los inicios de la década del 80, el régimen militar enfrentó una acelerada deslegitimación interna (Cavarozzi, 2002). El Gobierno de facto finalizó con la asunción, en diciembre de 1983, de Raúl Alfonsín -de la Unión Cívica Radical (UCR)- como presidente.

La dictadura estuvo encabezada por la Junta de Comandantes en Jefe, integrada por un representante de cada una de las Fuerzas Armadas, y contó con la complicidad de algunos sectores concentrados de la economía y del clero (Bilbao y Lede, 2016; Franco, 2016). La violencia ejercida y perpetrada por instituciones estatales en este período incluyó represión, secuestro, desaparición, tortura física y psicológica, asesinato y robo de niños y niñas (Bayer, Borón, Gambina, Barillaro y La Greca, 2010). En el plano económico, instauraron un sistema de libre mercado, que consistió en abrir el comercio interno a la competencia exterior (Cavarozzi, 2002).

8 Creado en 1972 por el decreto-ley 19798. 
María Eugenia Iturralde

Los medios audiovisuales del centro-sudeste bonaerense en el período 1983-2004

\section{Servicios audiovisuales en Azul, Olavarría y Tandil}

A continuación, se analizan algunas políticas de comunicación inscriptas en contextos sociopolíticos específicos, estableciendo relaciones con el desarrollo de procesos propios de las ciudades medias. Si bien el período abordado abarca de 1983 a 2004, para comprender algunos aspectos -sobre todo normativos- es necesario incorporar algunos datos de la etapa previa.

\section{Antes de 1983}

Ubicamos la apertura de la primera etapa de análisis en 1922, dado que un suceso dio inicio al desarrollo de los medios audiovisuales en las ciudades analizadas: la salida al aire de la radio perteneciente al diario Nueva Era en Tandil. La historia de la emisora es un signo de su época, ya que obtuvo dos autorizaciones para salir al aire, sin embargo, fue clausurada en 1931 y se puso en marcha de nuevo en 1932, hasta que sufrió otro cierre forzado por autoridades estatales el 25 de febrero de 1934, el cual incluyó el decomiso de lámparas y bobinas.

En el período que comprende desde la puesta en funcionamiento de la primera emisora radial hasta el final de la última dictadura militar en 1983, se registra la operativización de emisoras en $\mathrm{AM}$, señales locales de televisión por cable, una radio $\mathrm{FM}$ y el acceso a señales de canales de aire oriundas de Mar del Plata y de Capital Federal (Arabito, 1994; Iturralde, 2019). Todas estas iniciativas estuvieron inmersas en un contexto de profunda inestabilidad, en el que las acciones del Estado fueron interrumpidas y sustituidas por otras distintas al ritmo de los sucesivos Gobiernos constitucionales y de facto.

El Estado no adoptó de manera predominante el rol de prestador de los servicios, sino que asumió el lugar de controlador de las emisiones. Radio Olavarría y Radio Azul se crearon por iniciativa del Estado nacional, pero tiempo después fueron privatizadas. El sector privado fue el gran protagonista, imprimiendo una fuerte lógica comercial a los contenidos mediáticos producidos en el centro-sudeste bonaerense durante el siglo XX.

\section{De 1983 a 2004}

\section{El decreto-ley 22285 durante la presidencia de Raúl Alfonsín}

La llegada de Raúl Alfonsín al Poder Ejecutivo marcó un cambio de época plagado de expectativas vinculadas a la democratización de la sociedad argentina y particularmente de la comunicación. Durante su presidencia, el COMFER fue intervenido y se nombró a un delegado normalizador. Hasta ese momento, el directorio del organismo estaba integrado, entre otros, por representantes de las Fuerzas Armadas Argentinas. 
Austral Comunicación

Volumen 9, número 1 (Junio de 2020): 93-119. ISSN 2313-9129

Mediante el Decreto 1151 de 1984 se suspendió la aplicación del PLANARA y la convocatoria a sustanciar concursos públicos hasta la sanción de una nueva ley de radiodifusión.

En 1985, el PEN creó un organismo pluripartidario y multisectorial: el Consejo para la Consolidación de la Democracia (COCODE), que tenía entre sus objetivos primordiales el de avanzar en la redacción de una ley de radiodifusión.

A fines de 1987, se presentó el proyecto del COCODE para regular la radiodifusión, realizado con base en el análisis de propuestas existentes en la materia, reuniones de comisiones técnicas, legislación comparada y estudios sobre el tema. La publicación del proyecto estuvo acompañada de un prólogo que expresa lo siguiente:

Se llegó a la conclusión que (sic) la regulación de medios de radiodifusión debía asegurar
que ellos sirvieran tanto al enriquecimiento del debate colectivo como al desarrollo de la
autonomía individual y que para ello era necesario producir una gran apertura y expan-
sión del sistema existente; descentralizar los focos de distribución de licencias, dando por
primera vez activa participación a Provincias y Municipios; pluralizar las vías de acceso
al sistema, expandiendo el sector privado y comunitario y desdoblando el público en uno
gubernamental y otro en el que estén representados los grupos parlamentarios, las uni-
versidades y otras entidades educativas y culturales; evitar, aun con sanciones penales
para la simulación, que ningún grupo tenga una posición monopólica o dominante por
vía del control de múltiples medios de modo que su opinión prevalezca sobre las res-
tantes; velar porque consideraciones comerciales no se sobrepongan a consideraciones
de bien público, limitando el tiempo de publicidad y el contenido de publicidad dañina
para la salud física y psíquica promoviendo las expresiones artísticas de origen nacional,
asegurando espacios en que se traten cuestiones de interés social, previéndose el derecho
de rectificación de informaciones falsas y creándose una defensoría del público para ca-
nalizar los reclamos de los destinatarios de medios. (Consejo para la Consolidación de la
Democracia, 1988, p. 8).

El proyecto de ley estaba estructurado en 87 artículos y tuvo una vida fugaz, porque en abril de 1988 fue abandonado por el Gobierno nacional. Finalmente, la propuesta enviada al Congreso fue modificada y si bien desde el PEN no se explicaron las causas, los cambios sugieren que cedió ante las presiones de la Comisión Empresaria de Medios de Comunicación Independientes (CEMCI) y del diario Clarín (Com, 2006).

El decreto-ley 22285 continuó vigente y su articulado autorizaba a los titulares de servicios de radiodifusión en AM a optar por la instalación, en la misma localización, de una estación de FM. En ese sentido, el 21 de diciembre de 1984 comenzó a emitir en Olavarría la frecuencia modulada de LU 32, y el 25 de octubre de 1987 inició sus actividades la FM de LU 10 en Azul. ${ }^{9}$

En 1985, se lanza la televisión por cable en Tandil. Uno de los accionistas, Jesús Rocha, revela en una entrevista publicada en un diario de Tandil que la iniciativa surgió años antes en un retiro espiritual realizado en el Monasterio Trapense de Azul. La

Inicialmente denominada FM Celestial. 
María Eugenia Iturralde

Los medios audiovisuales del centro-sudeste bonaerense en el período 1983-2004

existencia del servicio de cable en Azul incentivó a un gran grupo de tandilenses a invertir en el mismo sentido en su ciudad (El Eco de Tandil, 2007). La firma Cerrovisión S.A. ${ }^{10}$ obtuvo permiso del COMFER para emitir (COMFER, 1985). Luego del período de prueba, inició sus transmisiones regulares el 15 de enero de 1986 (Cablevisión, 2015a), conteniendo en la grilla la señal local de Canal 2.

En 1986 y 1988 se modificó la composición accionaria de Radiodifusora Tandil S.R.L, con licencia desde 1968 e integrada originariamente por Osvaldo Edmundo Repetto (teniente coronel), Homero Alfredo Fortunato, Oscar José Romano (comodoro), Juan Carlos Derosa, Oscar Saliani y Alcides Santos Fortunato. En primer lugar, ingresó Alejandro Homero Fortunato; luego, se desvincularon Repetto, Romano, Derosa y Salian; y finalmente se incorporaron nuevos socios. ${ }^{11} \mathrm{La}$ emisora quedó bajo la dirección de Juan Vicente Martínez Belza.

La década del 80 se despidió dando lugar a un fenómeno que años después se desplegaría con gran potencia: la proliferación de radios en frecuencia modulada. Sin mediar permisos estatales para emitir, en el centro-sudeste bonaerense se encendieron los transmisores de las emisoras en FM. Cuando el mandato de Alfonsín estaba finalizando, un decreto autorizó a las universidades nacionales a instalar y operar servicios de radiodifusión sonora con modulación de frecuencia. El artículo 15 del Decreto 789 establece que la Universidad Nacional del Centro de la Provincia de Buenos Aires (UNICEN) tenía permiso legal para instalar y operar tres servicios de radiodifusión en Azul, Olavarría y Tandil. Paradójicamente, mientras titulares sin habilitación emitían, esa casa de altos estudios tardó veinte años en lanzar su emisora.

El Gobierno de la UCR se sostuvo hasta que sobrevino la explosión inflacionaria en 1989. Los errores del oficialismo resultaron altamente costosos para el mantenimiento del poder, situación que fue aprovechada por sus adversarios políticos.

Carlos Menem, del Partido Justicialista (PJ), asumió la presidencia anticipadamente el 8 de julio de 1989. Cavarozzi (2002) señala que hacia fines de la década del 80 la política se devaluó, dando lugar al aumento de posiciones en favor de la contracción drástica de las esferas de acción y regulación estatal. "El Estado se derrumbó a partir de 1981-1982 y, asimismo, se achicó desde 1990-1991 en adelante; tanto el derrumbe como el achicamiento han cambiado radicalmente la trama de la sociedad argentina" (Cavarozzi, 2002, p. 65).

\footnotetext{
${ }^{10}$ Integrada por Julio Baqueriza, Raúl Arana, Edgardo Carra, Alberto Iribarne, Julio Ochandorena, Luis Magnasco, Jesús Rocha, Alberto Barreira, Juan Carlos Pugliese (h), Juan Manuel Schang, Roberto Cardinale y otros.

${ }^{11}$ La renovación de la licencia se produjo con la siguiente composición de la sociedad: Juan Vicente Martínez Belza, José Julio Nachinowichz, Miguel Ángel Martínez, Alberto Ramón Martínez, Graciela Beatriz Grasso de Meli, Carlos Enrique Bader, José Manuel Peña, Marcelo Daniel Carrillo, Enrique Eduardo Aracil, Jorge Víctor Pellitero, Héctor Antonio Concetti, Obdulio Pablo Lora, Alejandro Homero Fortunato y Alcides Carlos Fortunato.
} 
Austral Comunicación

Volumen 9, número 1 (Junio de 2020): 93-119. ISSN 2313-9129

\section{El decreto-ley 22285 durante las presidencias de Carlos Menem}

Durante la primera presidencia de Menem (1989-1995), se reformó la Carta Magna, incluyendo modificaciones al sistema electoral. La reforma constitucional de 1994 hizo posible que Menem fuera reelecto como presidente (1995-1999). El objetivo central de estos Gobiernos fue estabilizar la economía, aplicando un programa contrario a los postulados nacionalistas y estatistas tradicionalmente apoyados por el PJ (Fair, 2009). El plan gubernamental incluyó el recorte del gasto público, la aplicación de restricciones a la oferta monetaria, el sostenimiento de niveles mínimos de inversión pública y la política cambiaria apuntó a estabilizar el dólar. ${ }^{12}$ Estas medidas profundizaron la recesión y generaron una fuerte caída de la producción industrial. Se privatizaron, disolvieron y concesionaron empresas y servicios públicos (Pucciarelli, 2011).

Oszlak (2003) analiza la reestructuración organizacional del Estado argentino en la década del 90 y sostiene que es preciso caracterizar ese proceso como una transmutación más que como una minimización. Durante ese período, el Estado modificó su fisonomía, su dominio funcional y su papel frente a la sociedad, pero no desapareció. El autor considera que el Estado renunció a cumplir ciertas funciones asociadas al bienestar de los sectores sociales pobres y a regular las actividades socioeconómicas, como tradicionalmente lo hacía. Asimismo, se desligó de algunas obligaciones, descentralizándolas a otros niveles territoriales y políticos. Por último, redefinió sus modalidades de intervención, destacándose su rol como recaudador central y principal asignador de los recursos a través del presupuesto de gastos consolidado.

En julio de 1989, asumió su primera presidencia Carlos Menem, en agosto del mismo año se sancionó la Ley 23696 de Reforma del Estado; y en septiembre, la Ley 23697 de Emergencia Económica. Guillermo Mastrini y Mariano Mestman (1996) consideraron la Ley de Reforma del Estado como un ejemplo de re-regulación, a diferencia de las conceptualizaciones que veían en la actuación gubernamental un proceso de desregulación. El argumento de la retirada del Estado y la apertura al libre mercado escondía una profunda adecuación normativa en pos de una nueva estructura de propiedad.

Sostenemos que el uso del concepto de desregulación constituye una falacia construida a partir de presentar Estados en retirada, cuando por el contrario esos Estados se encuentran en la primera línea de batalla, generando un volumen de dispositivos legales, en muchos casos mayor que los previos, destinados a establecer reglas de juego acordes con los intereses de los grupos oligopólicos" (Mastrini y Mestman, 1996, p. 82).

\footnotetext{
12 Las medidas económicas enumeradas responden al Consenso de Washington, diez fórmulas de "buen comportamiento" tomadas del economista John Williamson, las cuales los países deudores tenían que cumplir frente a organismos financieros con sede en Washington, como el Fondo Monetario Internacional, el Banco Mundial y el Departamento del Tesoro de los Estados Unidos. El Estado nacional tenía que achicarse para pagar la deuda externa, limitándose a cumplir con funciones básicas como el mantenimiento del orden y de la seguridad pública.
} 
La Ley de Reforma del Estado incluyó modificaciones al decreto-ley de Radiodifusión ${ }^{13}$ y se eliminaron varias de las restricciones que este establecía: el límite de tres licencias para una misma persona, presentarse a concurso con el fin de obtener un permiso siendo propietario o socio de diferentes sociedades de radiodifusión, que se presenten a concurso personas vinculadas a la propiedad de medios gráficos, que participen como licenciatarias personas con otras actividades económicas más allá de la explotación de servicios de radiodifusión y que las sociedades licenciatarias estén compuestas por menos de veinte personas físicas. Así, las medidas restrictivas para otorgar licencias, impuestas por el último Gobierno de facto, quedaron sin vigencia. La radiodifusión se abrió más al mercado, excluyendo a capitales extranjeros. Esa ley ordenó la privatización de L.S. 84 TV Canal 11, L.S. 85 TV Canal 13, L.R. 3 Radio Belgrano, L.R. 5 Radio Excelsior y todos los medios administrados por el Estado, excepto Canal 7, Radio Nacional Buenos Aires, Radio Difusión Argentina en el Exterior y las emisoras integrantes del Servicio Nacional de Radiodifusión.

El artículo 65 de la Ley de Reforma del Estado facultó al PEN a adoptar las medidas necesarias para regular el funcionamiento de aquellos medios que no se encuadraban en las disposiciones vigentes hasta ese momento. El texto original del decreto-ley 22285 no daba lugar a la participación de la Iglesia católica argentina en la radiodifusión, pero en 1990 el COMFER firmó la Resolución 858 para autorizarla a ser sujeto de servicios de radiodifusión, a diferencia de los medios sin fines de lucro que continuaron sin poder operar legalmente. Durante el segundo Gobierno de Menem, la Iglesia Católica, como persona jurídica de carácter público, ${ }^{14}$ se constituyó como el principal grupo de medios de comunicación por la cantidad de emisoras que tenía bajo su órbita en la totalidad del territorio nacional (Albornoz y Hernández, 2006). En 1993, el Decreto 311 autorizó al Obispado de Azul a instalar y operar un servicio de radiodifusión sonoro en la frecuencia 99.5 de Tandil, y por iniciativa del párroco Raúl Troncoso salió al aire FM Radio de la Sierra.

En términos generales la mayoría de los decretos y resoluciones que se implementaron en relación a la política de medios después de 1984 beneficiaron la concentración, el negociado y la manipulación de los medios masivos de comunicación en favor de empresas privadas, en detrimento de lo que pudo haber sido una comunicación con sentido comunitario (Com, 2006, p. 207).

La concentración es un proceso por el cual un número cada vez menor de capitalistas controla un mayor volumen de medios de producción y fuerza de trabajo, generando un aumento en el tamaño de sus empresas, más producción de mercancías $\mathrm{y}$ crecimiento de las tasas de rentabilidad. Esto implica que pocas manos controlan los

${ }_{13}$ En los artículos 43, inciso c; 45, inciso e; y 46, incisos a y c.

14 Establecido por el artículo 33, inciso 3 del Código Civil. 
medios de producción con los que cuenta una sociedad, posicionándose de manera ventajosa porque, además, imponen barreras para el ingreso de nuevos competidores al mercado (Loreti y Lozano, 2014). La concentración no es un fenómeno particular de Argentina, es una problemática latinoamericana (Becerra y Mastrini, 2017; Mastrini y Becerra, 2001; 2011). Los prestadores comerciales de servicios -que concentran la propiedad de los medios- no solo se benefician en el plano económico, sino que también obtienen rédito político. Es importante señalar que las acciones de los medios de comunicación intervienen, afectan y constituyen el espacio público, que es un espacio político. Los medios de comunicación son instituciones que poseen intereses económicos y políticos y trabajan con mercancías que tienen doble valor: material y simbólico (Mastrini y Becerra, 2011).

Damián Loreti (1995) señala que un ejemplo claro de concentración en medios de comunicación es la conformación de multimedios a inicios de la década del 90, generalmente constituidos por una empresa propietaria de un medio gráfico que accedió a la explotación de una radio AM con su FM complementaria y un circuito cerrado de televisión por cable o codificado que, eventualmente, sumaba la participación en agencias de noticias o proveedoras de papel. En Olavarría se observan procesos que confirman lo que menciona Loreti.

En primer lugar, la derogación del inciso e. del artículo 45 del decreto-ley 22285 legalizó la posibilidad de acceso a licencias de personas vinculadas a la propiedad de medios gráficos, situación legal que transparentó el vínculo existente entre El Popular S.A. y Teledifusora Olavarría S.A. En 1981, se comenzó a gestar en Olavarría la llegada de la televisión por suscripción. Julio Mario Pagano -director de El Popular- se reunió con Reynaldo Warman para transmitirle la intención de impulsar la televisión por cable. El COMFER aprobó los trámites por medio de la Resolución 171 del 29 de marzo de 1983. Formalmente, la sociedad comercial estaba compuesta por Joaquín Singla, Andrés Singla, Arturo Vercesi, Francisco Amoroso, Enzo Soccini y Reynaldo Warman. Este último fue el director del canal durante quince años y en un libro reveló que Pagano era el titular del canal, pero legalmente no figuraba porque era uno de los dueños de El Popular.

[...] Julio buscó alternativas, en conocimiento de que capitales porteños llegarían a nuestro medio para instalar un canal de televisión por cable, que se convertiría sin duda, en una preocupante competencia para el matutino. Amigos de su confianza integrarían la nueva empresa, invitándome a participar con ellos como accionista y director teniendo repartidas proporcionalmente las acciones del Grupo Pagano (Warman, 2014, p. 35).

Canal 5 de Olavarría comenzó a emitir en 1983 y formalmente sus transmisiones regulares datan del 25 de marzo de 1984 (Cablevisión, 2015b). 
En segundo lugar, en 1993, Amalia Lacroze de Fortabat ${ }^{15}$-accionista de LU 32 Radio Olavarría y su FM- adquirió el 51\% de las acciones del diario La Prensa, Radio El Mundo y FM Horizonte.

Otra medida favorable para la conformación y consolidación de multimedios fue la firma del Decreto 1771 de 1991, por medio del cual se modificó el artículo 68 del texto original del decreto-ley 22285. Esta normativa flexibilizó la prohibición para constituir redes de transmisión permanentes, ya que aprobó la integración de licenciatarios a una red de programación si, entre otras condiciones, se mantenían los derechos de publicidad de cada emisora y si las estaciones afiliadas originaban el 50\% de la emisión. En 1993, la FM de Radio Olavarría comenzó a transmitir en red la programación de FM Horizonte y adoptó el nombre que aún conserva: Cristal.

Como ya anticipamos, en la década del 90, las emisoras radiales en frecuencia modulada se multiplicaron. La tecnología y el equipamiento necesarios eran más baratos que los utilizados para transmitir en $\mathrm{AM}$, incluso se fabricaban equipos caseros (Kejval, 2009). De acuerdo a datos publicados por el COMFER, en 1998 operaban setecientas FM legales en Argentina y aproximadamente dos mil que no estaban empadronadas o no cumplían los requisitos legales vigentes. A principios de esta década, se entregaron Permisos Precarios y Provisorios, y en los años posteriores se dictaron decretos del PEN y Resoluciones del COMFER y de la Secretaría de Comunicaciones con el objetivo de normalizar el funcionamiento del espectro radioeléctrico (Albornoz y Hernández, 2006).

En 1992, emisoras FM de Olavarría fueron intimadas por el COMFER (Integración, Luz, Puerto Libre, Amanecer -de Loma Negra-, Master -de Sierras Bayas- y Bangkok), a diferencia de los responsables de FM Moebius y FM Success, que fueron citados por el organismo. De acuerdo con las publicaciones de El Popular, eran diez las emisoras locales al aire, pero solo dos estaban reconocidas por el ente estatal: Cristal -que poseía la licencia complementaria de la AM- y Asia, que tenía un Permiso Precario y

\footnotetext{
${ }^{15}$ La familia Pourtalé llegó de Francia a los actuales territorios del centro bonaerense en la primera mitad del siglo XIX. Los hijos de Jacques Pourtalé y Marie Hounau fundaron la estancia Las Cortaderas en lo que luego sería territorio azuleño, mientras que un familiar llamado Joaquín Pourtalé fundó en 1873 la estancia La Tigra, primer sitio alambrado (en 1881) del actual territorio de Olavarría. Uno de los residentes de la estancia azuleña contrató a un maestro francés, Luciano Fortabat, para la instrucción de las jóvenes de la familia. El educador contrajo matrimonio con Elena Pourtalé, unión de la que nació Alfredo Fortabat. El hijo más pequeño de la pareja Pourtalé-Fortabat heredó de su abuelo una parte de la Estancia San Jacinto y compró las que les correspondían a sus hermanos. El terrateniente cambió la denominación de las tierras por Estancia Fortabat. El descubrimiento de caliza y arcilla en la propiedad abrió un nuevo camino de inversiones que llevó a Alfredo a la apertura de la primera planta de Loma Negra S.A., en el año 1926 (Lemiez, 2016). En 1955, Alfredo se casó en segundas nupcias con una integrante de una familia aristocrática, María Amalia Sara Lacroze Reyes. Alfredo supo multiplicar la riqueza familiar de la mano de la industria, pero tras su muerte, en 1976, Amalia asumió la dirección de las empresas y logró posicionarse como la mujer más rica de Argentina. La familia Fortabat condensó la dimensión "benefactora" de los grandes empresarios (Sosa, 2016) y, estableciendo una relación paternalista (Fernández Massara, 2018), hicieron, sobre todo en Olavarría, múltiples donativos para la construcción de edificios de uso público (hospitales, instituciones educativas, caminos y templos católicos).
} 
Provisorio de 1990. ${ }^{16}$ En respuesta al COMFER, FM Integración presentó un recurso de amparo y el Juzgado Federal de Azul falló a su favor prohibiendo la incautación, el decomiso y la afectación de la salida al aire. Puerto Libre hizo lo mismo. En septiembre de 1993, visitó la ciudad Eduardo J. Lezcano, jefe de zona de la Dirección General de Fiscalización del COMFER, con el objetivo de informar a la población sobre la legalidad de las emisoras operativas en frecuencia modulada (El Popular, 1993). Success y Moebius contaban con el Permiso Precario y Provisorio. El 24 de abril de 1994, El Popular publicó una investigación especial en la que brindó detalles sobre la situación de las emisoras. El 12 de mayo de ese año, la Policía Federal (en presencia de una delegación de la Comisión Nacional de Telecomunicaciones) allanó varias radios -Mix, Luz, Bangkok y Master- a las que también se les incautaron equipos.

\begin{abstract}
Si bien se dio como supuesto que el trabajo de inteligencia de la Policía Federal olavarriense llevaba mucho tiempo, no se desconoció que la amplitud del operativo y la urgencia por actuar sobre el tema estuvo centrado en la nota de investigación que publicara El Popular el pasado 24 de abril. Allí se informó con lujo de detalles el caos en el que había entrado la zona a partir de la utilización masiva de equipos de comunicaciones aéreas sin permiso oficial ni homologación. (El Popular, 13 de mayo de 1994, p. 7).
\end{abstract}

En 1994, fue cancelada la inscripción precaria y provisional otorgada a favor de Walter Rubén Ciavarella para operar FM Moebius (COMFER, 1994a). El COMFER argumentó que la decisión era producto de la acreditación de graves incumplimientos al Decreto 1357 de 1989.

La llegada de las FM incomodó a los históricos sectores vinculados a los medios locales. Años antes, los medios operativos en la región instauraban redes colaborativas con corresponsalías. Al parecer, en Olavarría, la irrupción de nuevos actores sin vinculación al apellido Fortabat o a los accionistas de El Popular desató una feroz competencia con denuncias por ilegalidad ante los organismos públicos.

Al promediar la década del 90, a la concentración de la propiedad se le sumó otro fenómeno: una importante cantidad de infraestructura, medios, productos y mensajes empezaron a ser propiedad o a estar bajo control de agentes transnacionales. Durante la segunda presidencia de Carlos Menem, aumentó la presencia de capitales extranjeros que adquirieron las principales empresas de radiodifusión del país (Albornoz y Hernández, 2006).

En el año 1992, mediante la Ley 24124, entró en vigor el Tratado entre la República Argentina y los Estados Unidos de América sobre la Promoción y Protección Recíproca

\footnotetext{
${ }_{16}$ En 1989, el PEN firmó el Decreto 1357 con el objetivo de avanzar en la adjudicación de licencias de frecuencia modulada. Puntualmente, la normativa instauró la apertura de un registro de inscripción para personas físicas o jurídicas que operaron estaciones de radiodifusión sonora con modulación de frecuencia hasta el 17 de agosto de 1989, a las que posteriormente se les autorizó a operar, en forma precaria y provisional, sin conferirles derecho alguno en el futuro y sujeto a estrictas condiciones de cancelación automática.
} 
de Inversiones. ${ }^{17}$ Diego Rossi (2006) señala que la reciprocidad a la que refiere el documento no fue aplicable a empresas vinculadas a industrias culturales, ya que el país del norte las protegió de los alcances de este mediante un anexo. Las empresas argentinas no tenían permitido invertir en industrias culturales radicadas en Estados Unidos, pero los capitales estadounidenses sí podían hacerlo en nuestro país.

A la firma del tratado con Estados Unidos le siguieron acuerdos similares con Italia (Congreso de la Nación, 1992b), Francia (Congreso de la Nación, 1992a) y el Reino de los Países Bajos (Congreso de la Nación, 1996), entre otros. En materia de radiodifusión, los acuerdos entraban en conflicto con el decreto-ley 22285, puesto que la norma impedía la participación de capitales extranjeros en las empresas prestatarias de estos servicios. Pero la reforma de la Constitución Nacional de 1994 estableció que los tratados internacionales están jerárquicamente por encima de las leyes nacionales. De ese modo, se legalizó el ingreso de capitales estadounidenses al mercado argentino de radiodifusión.

En la segunda mitad de la década del 90, casi la totalidad de los principales medios de radiodifusión fueron transferidos a agentes vinculados, en su mayoría, al mercado financiero. A partir de 1994, se formalizaron relaciones entre empresas nacionales vinculadas a medios de comunicación y capitales extranjeros. Se conformaron alianzas, fusiones, ventas con operadores, bancos y fondos de financiamiento que permitieron afrontar inversiones para el tendido de fibra óptica y la renovación del equipamiento tecnológico. El Grupo Clarín, financiado por Citicorp, compró diversos cables operadores que pasaron a formar parte de Multicanal y Red Argentina S.A. Por otro lado, la compañía estadounidense TCI compró el 80\% de Cablevisión, y la empresa Continental, de la misma procedencia, el 50\% de Video Cable Comunicación (VCC) (Rossi, 2006).

A principios de esa década, existían en Argentina 1100 operadores de cable. El Estado no estableció zonas de exclusividad para las distribuidoras, por ese motivo, la competencia y las ofertas se multiplicaban. Pero, al finalizar el siglo XX, el número de empresas de cable había disminuido muchísimo: grandes operadoras las absorbieron frente a la expectativa que generaba la convergencia de las redes de televisión con futuros servicios de telecomunicación.

En 1990, estaban en actividad, en las ciudades analizadas, Azul TV Canal 2 S.A., Teledifusora Olavarría S.A., Cerrovisión S.A. y Video Cable Chillar. ${ }^{18}$ Ese año, se emi-

\footnotetext{
17 En 1995, el COMFER firmó la Resolución 350 en la que aprobó las condiciones y requerimientos que debían cumplir las personas, físicas y jurídicas que solicitaran la adjudicación de licencias para la instalación, funcionamiento y explotación de servicios de radiodifusión, en el marco del tratado con Estados Unidos aprobado por la Ley 24124 de 1992.

${ }_{18}$ Obtuvo el permiso para transmitir mediante la Resolución 795 de 1986 del COMFER.
} 
tió un permiso ${ }^{19}$ para que operara en Sierras Bayas un sistema codificado de televisión, SIBATECO S.A., que inició sus actividades el 28 de febrero de 1991 (Cablevisión, 2015c). En febrero de 1993, el Consejo Deliberante de Olavarría aprobó la solicitud, efectuada por Daniel Foti, para usar el espacio aéreo con el objetivo de operar un servicio de televisión por cable. La iniciativa no prosperó; Foti no hizo las presentaciones correspondientes en el COMFER porque fue acusado y detenido por efectuar actividades comerciales presuntamente ilegales. Tandil sí logró ampliar la oferta de cable. En octubre de ese año, comenzó a operar Megadifusora Tandil S.R.L. Asimismo, se entregaron permisos a Telesierra S.R.L (para operar en María Ignacia Vela), a Video Cable Tandil S.A. y a TV Tandil S.A.

En 1994, se registran actividades que contaron con la participación de los titulares de cables -algunas impulsadas por la Asociación Bonaerense de Televisión por Cable (ABT) - con el objetivo de desarrollar estrategias de unidad para optimizar las ganancias de las operadoras. Además, sostuvieron conjuntamente un fuerte cruce con el Gobierno provincial cuando el Poder Legislativo impuso un impuesto al cable. En respuesta, los canales establecieron un quite de cámara a los funcionarios en todo acto o entrevista de orden político en la provincia, no realizando coberturas. Consideraban que era injusto pagar un porcentaje de ingresos brutos, teniendo en cuenta que ya abonaban al COMFER un impuesto similar.

Los intentos para que las firmas adoptaran medidas conjuntas no prosperaron. Era difícil dejar a un lado la dinámica individual para inscribirse en acciones compartidas, sumado a un marco jurídico beneficioso para los actores internacionales y a las permanentes demandas tecnológicas que requerían una gran inversión monetaria (Marino, 2017b).

Frente a esta situación, la venta de las cableoperadoras locales se presentaba como una opción. Julio Baqueriza -accionista del cable de Tandil Cerrovisión S.A.- y Julio Gutiérrez -accionista de una empresa de cable de Necochea- no fueron seducidos por las ofertas de VCC y Cablevisión, entonces evaluaron otras alternativas. Al buscar inversiones en Estados Unidos, conocieron al abogado estadounidense Paul Salvodelli -vinculado a Hicks, Muse, Tate \& Furst (HMT\&F) $-{ }^{20}$ y formaron BGS Group. En noviembre de 1996, bajo la firma Mandeville Cable Partners Argentina, fusionaron 67 compañías de cable -entre las que se encontraban Teledifusora Olavarría, SIBATECO, Cerrovisión y Azul TV Canal 2-, las cuales sumaban 400000 abonados. Meses después, Mandeville fue adquirida por Cablevisión S.A. (Boerr, 1998).

Hacia fines de 1997, Multicanal, perteneciente al Grupo Clarín, y Cablevisión-TCI, del Grupo CEI-Telefónica, adquirieron VCC por partes iguales y meses después se

${ }_{19}$ Resolución 824 de 1990 del COMFER.

${ }^{20}$ Firma de capital privado registrada en Estados Unidos. 
María Eugenia Iturralde

Los medios audiovisuales del centro-sudeste bonaerense en el período 1983-2004

repartieron los abonados. Mediante esta transacción, accedieron al control de más del $70 \%$ de los cinco millones de abonados de todo el territorio nacional. Ya en 2000, el fondo de inversión estadounidense HMT\&F controlaba la totalidad de Cablevisión, mientras que pequeñas empresas cableoperadoras -aproximadamente quinientas- esparcidas por todo el país trataban de mantenerse activas afrontando negociaciones desventajosas con los programadores internacionales (Albornoz y Hernández, 2006).

Cablevisión, como titular de las empresas de cable de las ciudades analizadas, ofreció las señales televisivas a los tradicionales diarios locales. Así, Canal 5 de Olavarría adoptó la denominación de Canal Local y su administración quedó a cargo de $E l$ Popular; en 2000, Canal 2 de Tandil empezó a ser administrado por El Eco de Tandil y pasó a llamarse Eco TV. El matutino El Tiempo de Azul no aceptó la propuesta y Cablevisión asumió la administración de la vieja señal de Canal 2 con el nombre de Somos Azul.

La frecuencia modulada no paró de poblarse en el territorio nacional. Hacia 2000, existían 679 emisoras en FM legales, 195 bajo la administración del Estado en diversos niveles y el resto en manos de operadores privados. De acuerdo al Decreto 1646 de 1996, 59 emisoras de Radio Nacional integraban el Servicio Oficial de Radiodifusión. Durante las dos presidencias de Carlos Menem, se autorizaron a diversos agentes a prestar servicios radiofónicos: Ejército Argentino, Instituto Superior del Ejército, escuelas rurales de frontera, Policía Federal, policías de provincias y Gendarmería Nacional.

Entre octubre y diciembre de 1999, el COMFER, luego de efectuar planes técnicos, entregó 439 licencias de FM. Entre los adjudicatarios figuraban conglomerados como el Grupo Clarín y el Grupo UNO (Albornoz y Hernández, 2006). De acuerdo con el procedimiento administrativo iniciado por el organismo en 1999, en los años siguientes se entregaron licencias en el centro-sudeste bonaerense (tres en la ciudad de Azul, una en Chillar (partido de Azul), cinco en Olavarría y nueve en Tandil) para instalar, operar y explotar emisoras FM. En Tandil también se le otorgó permiso a Editorial La Capital S.A. para instalar una emisora AM. Mediante un expediente iniciado ese año, en 2005 Radiodifusora Tandil S.R.L. accedió a la licencia para operar FM Galáctica, operativa desde 1979 (Poder Ejecutivo Nacional, 2005).

Antes de finalizar su segundo mandato presidencial, Carlos Menem firmó el Decreto 1005 de 1999, instrumento que adecuó el plexo normativo de la radiodifusión a los intereses de los grandes grupos económicos, formalizando la situación de concentración y extranjerización que el mercado impuso durante los diez años anteriores. Se modificaron al menos diez artículos del decreto-ley 22285, por medio de los cuales se extendió el máximo de licencias de 4 a 24 por operador, se facilitó la transferencia de permisos, se explicitó el ingreso de capitales extranjeros provenientes de países con los que se firmaron tratados de promoción y protección de inversiones, se habilitó la 
Austral Comunicación

Volumen 9, número 1 (Junio de 2020): 93-119. ISSN 2313-9129

constitución de redes privadas permanentes y se amplió la pauta publicitaria por bloques de horarios.

\section{El decreto-ley 22285 durante la presidencia de Fernando de la Rúa}

El 10 de diciembre de 1999 asumió la Presidencia de la Nación Fernando de la Rúa, mandato que terminó con su renuncia el 21 de diciembre de 2001. Durante ese período, continuó vigente el modelo económico neoliberal, agravando la situación económica y el deterioro institucional del país.

Después de la asunción del nuevo mandatario, la Secretaría de Cultura y Comunicación suspendió todas las resoluciones por las cuales se habían adjudicado licencias para la instalación, funcionamiento y explotación de radios en el año 1999. El COMFER efectuó un proceso de revisión de las adjudicaciones que terminó en septiembre de 2000 y del que resultaron confirmadas 157 licencias. La suspensión al permiso otorgado a Editorial Capital para operar una AM en Tandil no fue levantada.

Las políticas de comunicación de este período oscilaron entre las propuestas emanadas del COMFER, que intentaban establecer avances en materia de radiodifusión, y las presiones desde otros estamentos del poder para mantener el statu quo (García Leiva, 2006). El COMFER desarrolló acciones tendientes a ordenar el espectro y la fiscalización de contenidos y en Tandil adjudicó una autorización a la Iglesia católica para operar una AM.

En 2001, Gustavo López, interventor del COMFER, impulsó un proyecto de ley de radiodifusión que fue presentado en abril en la Cámara de Diputados. Esa iniciativa no formó parte de una posición predominante en el Estado y por eso no logró superar la instancia de tratamiento en comisión. Ese año, también se modificaron aspectos dispuestos por el Decreto 310, con la finalidad de facilitar la normalización del espectro radioeléctrico para el funcionamiento de emisoras de frecuencia modulada por medio de mecanismos de concurso público y adjudicación directa en un contexto de transparencia. Días después, la Resolución 1138 del COMFER autorizó el llamado a concurso público para la adjudicación de licencias para la instalación, funcionamiento y explotación de cien estaciones de radiodifusión sonora por modulación de frecuencia, entre las que se encontraban la 94.1 de Azul, la 98.9 de Olavarría y la 100.1 de Tandil. Esos permisos no fueron entregados y en diciembre el Gobierno terminó abruptamente su mandato. 
María Eugenia Iturralde

Los medios audiovisuales del centro-sudeste bonaerense en el período 1983-2004

\section{Del estallido social a los 21 puntos por la emocratización de la radiodifusión}

De la Rúa abandonó la Casa Rosada en medio de una crisis no solo económica, sino también social y política que incluyó saqueos, estado de sitio, represión, cacerolazos y muertes en manos de las fuerzas de seguridad. A la renuncia le siguió la sucesiva asunción de cuatro presidentes en doce días: Ramón Puerta, Adolfo Rodríguez Saá, Eduardo Camaño y Eduardo Duhalde. Este último gobernó desde el 2 de enero de 2002 hasta el 25 de mayo de 2003, día en que asumió Néstor Kirchner.

En los años posteriores a la crisis de 2001, la agenda pública y la gubernamental tuvieron como tema central las problemáticas sociales ocasionadas por la situación económica. De 2002 a 2004 no se registran -en documentos oficiales ni publicaciones periodísticas- actos administrativos o acciones estatales tendientes a modificar los sistemas de medios de Azul, Olavarría y Tandil.

El estallido social provocó una fuerte devaluación de la moneda argentina, suceso que afectó directamente a los medios de comunicación. Muchas de las empresas del rubro habían contraído deudas en dólares durante la década del 90, a las que debían responder con ingresos menguantes y en pesos. Los sistemas de televisión por cable se vieron fuertemente afectados por el contexto, ya que tenían costos fijos en dólares y una gran carga impositiva, además, la crisis conllevó a la disminución de abonados al servicio. En octubre de 2001, mediante un decreto presidencial, se bajó la alícuota establecida por la Ley de Radiodifusión.

Frente a esta situación, el Gobierno de Duhalde impulsó una normativa que luego fue aprobada en la gestión de Kirchner: la Ley 25750 de Preservación de Bienes y Patrimonios Culturales. Becerra y Mastrini (2016) entienden que esta legislación fue un salvataje estatal a las empresas de medios de comunicación. El texto de la norma fijaba un tope del $30 \%$ a la participación del capital accionario de empresas extranjeras, impedía que acreedores externos reclamaran como parte de pago los activos de las empresas nacionales endeudadas y limitaba su derecho a voto.

El gran beneficiado de la Ley de Preservación de Bienes y Patrimonios Culturales y de la sanción del Decreto 527 de $2005^{21}$ fue el Grupo Clarín, ya que evitó ser expropiado y, además, las licencias bajo su poder se revalorizaron.

[...] el Grupo Clarín logró sobrevivir tanto por los resultados de sus tácticas para el sector (reducción de costos, recaptura y fidelización de clientes) como por los beneficios obtenidos de las decisiones de políticas públicas (la otra cara de su estrategia) (Marino, 2017b, p. 111).

Si bien queda fuera del período de análisis, resulta importante señalar que hacia 2005 se inició el proceso por medio del cual el Grupo Clarín logró dominar el mer-

${ }^{21}$ Suspendió por una década el conteo de los años de las licencias de los servicios de radiodifusión. 
cado de las cableras. En 2007, mediante un dictamen de la Secretaría de Defensa de la Competencia, posteriormente rubricado por Néstor Kirchner, se habilitó la fusión de Multicanal y Cablevisión (Marino, 2017b). Las tradicionales empresas de cable del centro-sudeste bonaerense, nacidas como proyectos locales, fueron integradas a un oligopolio.

El período de análisis se cierra en 2004, ya que entendemos que en las ciudades analizadas comenzaron a gestarse proyectos disruptivos frente a la imperante e histórica lógica comercial. Ese año, al cumplirse 21 años de la reinstauración de la democracia, el Foro Argentino de Radios Comunitarias (FARCO) convocó a diferentes organizaciones de la sociedad civil interesadas en elaborar una nueva ley de radiodifusión que reemplazara la que estaba vigente. Esta última era considerada, por un lado, obsoleta jurídica y tecnológicamente y, por otro, ilegítima por llevar las firmas de funcionarios de un Gobierno de facto. De las reuniones surgió la redacción de los 21 Puntos Básicos por el Derecho a la Comunicación, documento que exigía la sanción de una nueva norma que garantizara el pluralismo informativo y cultural y recuperara reclamos de larga data. Tiempo después, ese grupo organizado formó la "Coalición por una Radiodifusión Democrática”. Los 21 puntos fueron presentados el 27 de agosto -Día Nacional de la Radiodifusión- de ese año, en un programa radial transmitido a todo el país desde el auditorio de Radio Nacional. Ese documento sentó las bases para la redacción de la Ley de Servicios de Comunicación Audiovisual de 2009.

\section{Conclusiones}

A mediados de la década del 70, se registró la caída de la matriz estado-céntrica. El Golpe de Estado de 1976 marcó el inicio de una etapa que, además de establecer un régimen político no democrático, sentó las bases del modelo económico que estallaría 25 años después. La instauración del neoliberalismo corrió al Estado de funciones asignadas tradicionalmente y permitió que los mercados internacionales operaran en el país casi sin intermediaciones.

El contexto político y económico de las décadas posteriores al último Gobierno de facto resultó propicio para la re-regulación, proceso mediante el cual se privilegiaron las actividades comerciales en materia de comunicación y se dejaron al margen a los medios sin fines de lucro, considerados ilegales. En la década del 90, la lógica comercial se impuso en un contexto de aplicación de políticas gubernamentales neoliberales y creció al ritmo de las modificaciones aplicadas al decreto-ley 22285, las cuales dieron lugar a la creación de grupos multimedios y a la concentración de la propiedad de los medios.

Los grupos concentrados de medios llevaron adelante acciones económicas y políticas tendientes a favorecer sus intereses (materiales y simbólicos). A la concen- 
María Eugenia Iturralde

Los medios audiovisuales del centro-sudeste bonaerense en el período 1983-2004

tración de la propiedad se le sumó que una importante cantidad de infraestructura, medios, productos y mensajes empezaron a ser propiedad o a estar bajo el control de agentes transnacionales. Durante la segunda presidencia de Carlos Menem, aumentó la presencia de capitales extranjeros que adquirieron gran parte de las principales empresas de radiodifusión del país.

Hacia fines del siglo XX, las manifestaciones populares públicas eran constantes, los sectores excluidos por el modelo económico neoliberal -introducido por el último Gobierno de facto y profundizado por el sistema de gobierno de Menem- permanecían en las calles expresando su situación de precariedad. Ya casi finalizando 2001, el conflicto social y la crisis institucional llevaron a la renuncia del presidente De la Rúa.

El desarrollo de los medios de comunicación en las ciudades medias en el período 1983-2004 siguió el ritmo sostenido por las acciones gubernamentales a nivel nacional. Durante el Gobierno de Alfonsín, se consolidaron y surgieron iniciativas privadas en televisión por cable y en frecuencia modulada. Si bien el Poder Ejecutivo no avanzó en la sanción de un marco normativo integral bajo un gobierno constitucional, llevó adelante algunas acciones democratizadoras para el uso del espectro radioeléctrico, como por ejemplo la entrega de autorizaciones para operar radios FM a la UNICEN, iniciativa que completó su objetivo más de dos décadas después con la salida al aire de Radio Universidad.

Como ya señalamos, las políticas de comunicación implementadas por los Gobiernos de Menem beneficiaron al sector privado. En el centro-sudeste bonaerense no se registraron avances de proyectos sin fines de lucro y en lo que respecta al sector público, gubernamentalmente se dio lugar al desarrollo de experiencias de la Iglesia católica argentina. La concentración de la propiedad de medios se evidencia en la conformación de un multimedio del grupo Fortabat, al que se integró AM Radio Olavarría y FM Cristal. Esta última comenzó a transmitir en red FM Horizonte, de la Ciudad de Buenos Aires, restándole horas de producción local a la grilla de la emisora. En ese momento, también surgieron los grupos de medios locales: El Popular Medios en Olavarría y El Eco Multimedios en Tandil. En el caso de El Popular, las modificaciones al decreto-ley 22285 permitieron regularizar y hacer pública la vinculación existente desde 1983 entre el servicio de cable y el diario.

A inicios de la década del 90, se multiplicaron las emisoras en frecuencia modulada en el centro-sudeste bonaerense, algunas -un número muy reducido- obtuvieron Permisos Precarios y Provisorios para operar. Otras salieron al aire durante años sin ningún tipo de acreditación, situación que las exponía a un peligro permanente. En Olavarría, en el año 1994, se decomisaron equipos y se clausuraron emisoras consideradas clandestinas por el Estado nacional, el mismo nivel estatal que hasta el año 1999 
Austral Comunicación

Volumen 9, número 1 (Junio de 2020): 93-119. ISSN 2313-9129

no concursó licencias en Azul, ni en Olavarría ni en Tandil para regularizar el funcionamiento de los servicios en frecuencia modulada.

No solo la concentración de la propiedad de medios de comunicación llegó al centro sudeste bonaerense, la extranjerización también se hizo presente. Los servicios de señal televisiva de pago, operados desde la década del 80 por personas jurídicas integradas por personas físicas residentes en Azul, Olavarría y Tandil, fueron transferidos a una firma de capitales estadounidenses hacia fines de los 90 .

En sintonía con el contexto nacional, en las ciudades medias analizadas existió un fuerte desarrollo de medios de gestión privada frente a un dispar impulso de experiencias estatales; se entregaron licencias a una universidad nacional -que no logró operativizar las emisoras- y a la Iglesia católica argentina, que sacó al aire una radio en amplitud modulada y otra en frecuencia modulada. En el período 1983-2004, se observa una fuerte alianza entre el Estado y el mercado, que se hace explícita en la aplicación de políticas de comunicación en beneficio de los fines de lucro de este último. Los poderes estatales no avanzaron en la sanción de una ley integral referida a medios de comunicación a causa de la resistencia del empresariado del sector. Tampoco se autorizó a las organizaciones sin fines de lucro a prestar servicios de radiodifusión.

\section{Referencias}

Albornoz, L. y Hernández, P. (2006). La radiodifusión en Argentina entre 1995 y 1999: concentración, desnacionalización y ausencia de control público. En Mastrini, G. (Ed.), Mucho ruido, pocas leyes. Economía y políticas de comunicación en la Argentina (1920-2004) (pp. 257-286). Buenos Aires: La Crujía.

Arabito, J. (1994). Un horizonte de antenas. El caso de la televisión en Olavarría (Tesis de grado). Facultad de Ciencias Sociales, Universidad Nacional del Centro de la Provincia de Buenos Aires.

Badillo, A., Mastrini, G. y Marenghi, P. (2015). Teoría crítica, izquierda y políticas públicas de comunicación: el caso de América Latina y los gobiernos progresistas. Comunicación y Sociedad, (24), 95-126. Obtenido de http://www.scielo.org.mx/ scielo.php?script=sci_arttext\&pid=S0188-252X2015000200005.

Bayer, O., Borón, A., Gambina, J., Barillaro, E. y La Greca, F. (2010). El Terrorismo de Estado en la Argentina. Buenos Aires: Instituto Espacio para la Memoria.

Becerra, M. (2014). Medios de comunicación: America Latina a contramano. Nueva Sociedad, (249), 61-74. Obtenido de http://nuso.org/articulo/ medios-de-comunicacion-america-latina-a-contramano.

Becerra, M. (2015). De la concentración a la convergencia. Políticas de medios en Argentina y América Latina. Buenos Aires: Paidós.

Becerra, M. y Mastrini, G. (2016). Políticas de medios del kirchnerismo. Análisis de 
las políticas de comunicación 2003-2015 y agenda pendiente. Análisis, (13), 1-26. Obtenido de https://library.fes.de/pdf-files/bueros/argentinien/12821.pdf.

Becerra, M. y Mastrini, G. (2017). La concentración infocomunicacional en América Latina (2000-2015). Nuevos medios y tecnología, menos actores. Buenos Aires: Universidad Nacional de Quilmes.

Bilbao, Lucas y Lede, Ariel (2016). Profeta del genocidio. El vicariato castrense y los diarios del obispo Bonamín en la última dictadura. Avellaneda: Sudamericana.

Bizberge, A. (2015). La regulación sobre medios audiovisuales en el siglo XXI en Argentina y Brasil. Revista Mexicana de Opinión Pública, (19), 13-39. Obtenido de https://www.sciencedirect.com/science/article/pii/S1870730015000022.

Boerr, M. (28 de mayo de 1998). Cambio de manos en los grandes negocios. El grupo Hicks quiere comprar en todo el país. La Nación. Obtenido de https://www. lanacion.com.ar/98067-el-grupo-hicks-quiere-comprar-en-todo-el-pais.

Cablevisión. (2015a). Planilla Anexo I de la carpeta de acceso público de la operadora de cable de Tandil. Obtenido de https://www.cablevisionfibertel.com.ar.

Cablevisión. (2015b). Planilla Anexo I de la carpeta de acceso público de la operadora de cable de Olavarría. Obtenido de https://www.cablevisionfibertel.com.ar.

Cablevisión. (2015c). Planilla Anexo I de la carpeta de acceso público de la operadora de cable de Sierras Bayas. Recuperado de https://www.cablevisionfibertel.com.ar.

Califano, B. (2015). Perspectivas conceptuales para el análisis del Estado y las políticas de comunicación. Austral Comunicación, 4(2), 251-286. Obtenido de http://www. austral.edu.ar/ojs/index.php/australcomunicacion/article/view/135).

Cavarozzi, M. (2002). Autoritarismo y democracia. Ciudad de Buenos Aires: EUDEBA

Com, S. (2006). Alfonsinismo, contexto sociopolítico y medios de comunicación. En Mastrini, G. (Ed.), Mucho ruido, pocas leyes. Economía y políticas de comunicación en la Argentina (1920-2004) (pp. 185-210). Buenos Aires: La Crujía.

Comité Federal de Radiodifusión. (COMFER). (1985). Resolución 15.

Comité Federal de Radiodifusión. (COMFER). (1994). Resolución del COMFER 878.

Congreso de la Nación. (14 de julio de 1992a). Ley, 24100, B.O. 27428. Obtenido de https://www.boletinoficial.gob.ar.

Congreso de la Nación. (25 de septiembre de 1992b). Ley 24122, B.O. 27480. Obtenido de https://www.boletinoficial.gob.ar.

Congreso de la Nación. (29 de agosto de 1994). Ley 24352, B.O. 27963. Obtenido https://www.boletinoficial.gob.ar.

Consejo para la Consolidación de la Democracia.(1988). Radiodifusión. Proyecto y Dictamen del Consejo para la Consolidación de la Democracia. Buenos Aires: EUDEBA.

El Eco de Tandil. (30 de julio de 2007). 125 Aniversario. Edición Especial.

El Popular. (10 de septiembre de 1993). Inspección del COMFER a radio de Olavarría. 
Austral Cmunicación

Volumen 9, número 1 (Junio de 2020): 93-119. ISSN 2313-9129

El Popular. (13 de mayo de 1994). La Policía Federal arrasó con radios y remises, Olavarría.

Exeni, J. (2010). Mediocracia de alta intensidad. Bolivia: Medios de comunicación y democracia en contextos de cambio. La Paz: IDEA Internacional.

Fair, H. (2009). Una revisión crítica de los estudios sobre el menemismo. Estudios. Revista del Centro de Estudios Avanzados, 21, 105-129. Obtenido de http:// biblioteca.clacso.edu.ar/Argentina/cea-unc/20101025014314/ESTUDIOS21.pdf.

Fernández Massara, M. (2018). Las mediaciones tecnológicas: tramas estructuralsimbólicas del orden institucional. En Lago Martínez, S., Álvarez, A., Gendler, M. y Méndez, A. (Eds.), Acerca de la apropiación de tecnologías: teoría, estudios y debates (pp. 153-165). Buenos Aires: Red de Investigadores sobre Apropiación de Tecnologías, Instituto Gino Germani. Rada Tilly, Gato Gris.

Franco, M. (2016). La noción de "dictadura cívico-militar". En Flier, P. (Coord.), Trabajos, Comunicaciones y Conferencias. Mesas de las VII Jornadas de Trabajo sobre Historia Reciente. La Plata: Facultad de Humanidades y Ciencias de la Educación, Universidad Nacional de La Plata.

García Leiva, M. (2006). Fin de milenio: concentración, continuidad y control. Una mirada sobre las políticas de radiodifusión del gobierno de Fernando de la Rúa. En Mastrini, G. (Ed.), Mucho ruido, pocas leyes. Economía y políticas de comunicación en la Argentina (1920-2004) (pp. 287-307). Buenos Aires: La Crujía.

Graziano, M. (1988). Política o ley: debate sobre el debate. Espacios, (7), 11-14.

Hernández Prieto, M. y Pérez Alaejos, M. (2017). Análisis del proceso de elaboración, sanción e implementación de la Ley de Servicios de Comunicación Audiovisual 26.522. El desinfle de un ideal. Communication \& Society, 30(2), 131-147. Obtenido de https://www.unav.es/fcom/communication-society/en/articulo. php?art_id=624.

Iturralde, M. (2019). Políticas de comunicación en ciudades intermedias. El proceso de discusión, sanción y aplicación de la Ley de Servicios de Comunicación en Azul, Olavarría y Tandil. La Plata: Universidad Nacional de La Plata. Obtenido de http:// sedici.unlp.edu.ar/handle/10915/73762.

Kejval, L. (2009). Truchas. Los proyectos políticos-culturales de las radios comunitarias, alternativas y populares. Buenos Aires: Prometeo.

Lemiez, G. (2016). No todo lo que brilla es cemento: relaciones laborales y conflicto en la industria cementera de Olavarría. En Gravano, A., Silva, A. y Boggi, S. (Ed.), Ciudades vividas. Sistemas e imaginarios de ciudades medias bonaerenses (pp. 275299). Buenos Aires: Café de las Ciudades.

Loreti, D. (1995). El Derecho a la Información. Relación entre medios, público y periodistas. Buenos Aires: Paidós. 
María Eugenia Iturralde

Los medios audiovisuales del centro-sudeste bonaerense en el período 1983-2004

Loreti, D. y Lozano, L. (2014). El derecho a comunicar. Los coflictos en torno a la libertad de expresión en las sociedades contemporáneas. Buenos Aires: Siglo XXI.

Marino, S. (2017a). Las políticas de cine en las industrias culturales argentinas. Chasqui. Revista Latinoamericana de Comunicación, (136), 357-374. Obtenido de https:// revistachasqui.org/index.php/chasqui/article/view/2766/2994.

Marino, S.(2017b). Políticas de comunicación del sector audiovisual: modelos divergentes, resultados equivalentes. La televisión por cable y el cine en la Argentina (1989-2007). Buenos Aires: Universidad Nacional de Quilmes.

Mastrini, G. y Becerra, M. (2001). 50 años de concentración de medios en América Latina: del patriarcado artesanal a la valorización en escala. En Quirós Fernández, F. y Sierra Caballero, F. (Eds.), Globalización, comunicación y democracia. Crítica de la economía política de la comunicación y la cultura. Sevilla: Comunicación Social Ediciones y Publicaciones.

Mastrini, G. y Becerra, M. (2011). Estructura, concentración y transformaciones en los medios del Cono Sur latinoamericano. Comunicar, 18(36), 51-59. Obtenido de https://www.revistacomunicar.com/index. php? contenido=detalles\&numero=36\&articulo $=36-2011-07$.

Mastrini, G. y Mestman, M. (1996). ¿Desregulación o re-regulación?: De la derrota de las políticas a las políticas de la derrota. CIC. Cuadernos de Información y Comunicación, 2, 81-88. Obtenido de https://dialnet.unirioja.es/servlet/ articulo? codigo $=188580$.

O Donnell, G. (2010). Democracia, agencia y estado. Buenos Aires: Prometeo.

Postolski, G. y Marino, S. (2006). Relaciones peligrosas: los medios y la dictadura entre el control, la censura y los negocios. En Mastrini, G. (Ed.), Mucho ruido, pocas leyes. Economía y políticas de comunicación en la Argentina (1920-2004) (pp. 159-188). Buenos Aires: La Crujía.

Oszlak, O. (2003). El mito del estado mínimo: una década de reforma estatal en Argentina. Desarrollo Económico, 42(168), 519-543.

Oszlak, O. (2007). Políticas públicas, democracia y participación ciudadana. Voces del Sur, (4), 8-11.

Oszlak, O.y O'Donnell, G. (1995). Estado y políticas estatales en América Latina: hacia una estrategia de investigación. Redes, 2(4), 99-128.

Poder Ejecutivo Nacional. (23 de junio de 2005). Decreto 706, B.O. 30680. Obtenido de https://www.boletinoficial.gob.ar.

Pucciarelli, A. (2011). Los años de Menem: la construcción del orden neoliberal. Buenos Aires: Siglo XXI.

Rossi, D. (2006). La radiodifusión entre 1990-1995: exacerbación del modelo privado- 
Austral Comunicación

Volumen 9, número 1 (Junio de 2020): 93-119. ISSN 2313-9129

comercial. En Mastrini, G. (Ed.), Mucho ruido, pocas leyes. Economía y politicas de comunicación en la Argentina (1920-2004) (pp. 235-256). Buenos Aires: La Crujía. Sosa, R. (2016). La educación que necesita la ciudad reconvertida. En Gravano, A., Silva, A. y Boggi, S. (Ed.), Ciudades vividas. Sistemas e imaginarios de ciudades medias bonaerenses. Buenos Aires: Café de las Ciudades.

Warman, R. (2014). 80 veces gracias. Creer. Crear. Crecer. Olavarría: Impacto. 